\title{
High Imperceptible Data Hiding using Remainder Method
}

\author{
Biswajita Datta \\ Assistant Professor, CSE \\ St. Thomas College of \\ Engineering \& Technology, \\ Kolkata, India
}

\author{
Samir K. Bandyopadhyay \\ Professor, CSE \\ CSE Department, \\ University of Calcutta, \\ Kolkata, India
}

\author{
Anurag Kedia \\ CSE \\ St. Thomas College of \\ Engineering \& Technology, \\ Kolkata, India
}

\begin{abstract}
In this paper we will propose a high quality image steganography technique which can hide secret data within an image in such a way so that it is imperceptible to human eye. This method solves the overlapping problem of remainder and use modulo operator efficiently for hiding purpose. First, we calculate the base eight equivalent of target string. That increases the hiding capacity as well as robustness of the stego image. Then the secret data can be hidden by remainder method. In our proposed work we alter the remainder of pixels in such a way so as to greatly reduce the image distortion caused by the hiding of the secret data. Experimental result checks the quality of stego image as well as compared with standard LSB technique and 3 LSB multi-bit image steganography technique. Experimental results have also demonstrated that the proposed scheme is secure against StirMark Benchmark 4.0.
\end{abstract}

\section{General Terms}

Security, Internet, Cryptography, Communication, Number System

\section{Keywords}

Steganography, Embedding, Extraction, Remainder method, Pre-processing, Post-Processing

\section{INTRODUCTION}

Today Security becomes an important issue with the rise of Internet. Cryptography plays an important role in this area for a decade. It focuses on keeping the contents of a message secret whereas now the modern scientists are focuses on keeping the existence of a message secret [1]. Steganography is the security technique for invisible communication. Steganography defines as "covered writing" [2]. Both Steganography and cryptography are not alone perfect. Once the presence of hidden information is suspected, the purpose of steganography is partly defeated. Thus the strength of steganography can be amplified by combining it with cryptography [3].

Although the concept of steganography is used for a long decade but do not have any name. Hidden on the back of wax writing tables, written on the stomachs of rabbits or Tattooed on the scalp of slaves, invisible writing by the help of invisible ink at the time of World War II are the early use of Steganography [4]. In the digital world of today, namely 1992 to present, Steganography is being used all over the world on computer systems. Steganography should meet three challenges: Imperceptibility -unnoticeable to human eye. Robustness - survival capability when the host medium has been manipulated. Capacity -embed large amount of data without much distortion [5].
The information can be embedded within different cover media like text, audio, image video etc. Image Steganography is one mostly used steganography technique. Here image is considered as a cover media and target information is embedded within that cover media to form a stego image [6]. Image steganography techniques are worked in both spatial and transform domain. There are currently three popular methods applied in Image Steganography: LSB Substitution, Blocking, and Palette Modification [7]. Now-a-days image steganography become popular with region selection for hiding based on the limited power of the human visual system (HVS) [8].

\section{LITERATURE SURVEY}

For a very long decade there are many steganography technique works for securing the Internet. Among different available techniques Least Significant Bit (LSB) insertion is a common and simple approach to embed information in an image file (Katzenbeisser, 2000) [9]. In this method the LSB of a byte is replaced with a bit of the secret message [10]. An optimal LSB substitution technique based on genetic algorithm is proposed by Wang et al. to improve the stegoimage quality [11]. Selected least significant bit steganography change one of the pixel color components in the image according to the message's bits to hide. The rest of bits in the pixel color component are also changed in order to get the nearest color to the original one in the scale of colors [12].

LSB embedding can be easily extended to hiding data in multiple bit-planes. B. R. Kumar et al. propose a new steganography technique for hiding target data in lowest 3 or 4 Least significant Bit-planes without any loss of data [13]. B. C. Nguyen et al. introduces a popular multi bit-plane image steganography technique [14]. Also a 3 LSB insertion method is used by A. Tripathi, J. S. Nair in their proposed work for embedding on maximum color used in histogram of the mosaic image [15]. A good payload-distortion steganography algorithm is introduced Zhang et al. Where the original palette is not changed and the colors in the palette are assigned to carry several bits [16]. But the major disadvantage of this kind of higher LSB Layer embedding is the reduction of perceptual transparency of stego image. Kawaguchi and Eason try to solve this problem by developing the bit-plane complexity segmentation (BPCS) steganography. Images are considered according to the different bit- planes and then the target bitplane for embedding is chosen by some threshold value [17].

Thien and Lin presents a simple LSB scheme based on the modulus function to improve the stego-image quality [18]. C. M. Wang et al. propose a steganography technique by using modulus operator. In their work the hiding capacity of the two consecutive pixels depends on the difference value of their intensity calculated by pixel-value differencing (PVD). Then the secret data is embedded by calculating the modulus of 
these consecutive pixels [19]. To give higher payload Wang has proposed also two new schemes based on modulo operator. A novel mechanism using modulus operations for embedding secret data into a cover image is proposed by Wang, S.J. They use modulus operation for selecting threshold that indicates how many bits of the secret image are being incorporated into the cover image [20].

\section{PROPOSED METHOD}

The remainder method has a disadvantage of overlapping. In this method the remainder is obtained for different values of data when divided by the same number. For example 13/8 \& $21 / 8$ have same remainder of 5 . So this remainder method cannot be used for hiding data in steganography due to large distortion. Also the remainder of a number cannot map to its exact dividend at the receiver side. That's why some of the target data is lost when try to extract it from stego media. We try to solve this problem in our proposed work.

For solving the overlapping problem of remainder here the encoding process is divided in different steps. First convert target data to the base of octal then hide these octal digits as information under cover image. In this paper text is considered as target data and 8 bit gray scale image as cover media. Both encoding and decoding techniques are discussed below.

\subsection{Data embedding}

The whole procedure for encoding is divided into three parts: Preprocessing of target data, embedding of target data into the cover media and the technique for storing the target string length.

\subsubsection{Preprocessing}

The length of text should be multiple of 3 for preprocessing of target data. If it is not the multiple of 3 then least used special character is concatenated at the end of the target string to make its length as multiple of three.

For preprocessing every three character is cut from the input string, and then converted their ASCII into 7 bit binary. Then concatenate them to form 21 bit string and cut every 3 bits from this string to convert them to their octal equivalent.

Let the string be "Image". The length of this string is 5 which is not multiple of three, add "" at the end of the string to make its length 6 which is now multiple of three. Now the following steps are performed for explaining the above process.

First the string is divided with 3 character each 'Ima', 'ge'. Take 1st three characters i.e. 'Ima".

ASCII of 'I', 'm', 'a' are 73, 109, and 97 respectively.

Now these are converted in 7 bit binary i.e. '1001001', '1101101' and ' 1100001 '

Then they are concatenated to form 21 bit string i.e. '100100111011011100001'

Now this string is divided by three bit each and converted them into decimal to get 7 octal digits i.e.

100100111011011100001

\section{$\begin{array}{lllllll}4 & 4 & 7 & 3 & 3 & 4 & 1\end{array}$}

The same above procedure also applicable for the next three

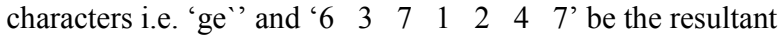
seven digit octal string.
After the above transformation " $4 \begin{array}{llllllllllll}4 & 7 & 3 & 3 & 4 & 1 & 6 & 3 & 7 & 1 & 2\end{array}$ 4 7" become target data. Now these octal digits are embedded into the cover image starting from the second row using remainder method. The embedding technique is discussed below.

\subsubsection{Embedding using remainder method}

During embedding the intensity of cover image pixels are divided by 8 , and their remainders are taken. Then these remainders are compared with each octal digit. If they equal then the pixel value is unaltered otherwise pixel value is adjusted in such a way so that its remainder is equal to the octal digits.

Now let the segment of cover image matrix be

$\mathrm{A}=\left[\begin{array}{lllllll}\ldots & 23 & 44 & 66 & 55 & 77 & \ldots\end{array}\right.$

$\begin{array}{lllllllll}56 & 77 & 12 & 33 & 23 & 66 & 77 & 22 & \ldots\end{array}$

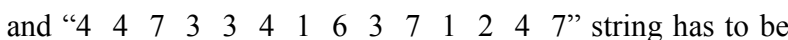
embedded as target string into this cover image matrix. For explanation 1 st value from the cover image segment is considered, then perform its modulus i.e. $\bmod (23,8)=7$. The 1 st digit from the target octal string is 4 which is not equal to 7. So it needs to adjust the pixel value to make its remainder equal to 4 .

There are large numbers within 0 to 255 whose remainder is 4 (which are 28, 4, 12, 20 and many more). But if we replace 23 by any one of these values, creates huge distortion and we are fails to meet the challenges of steganography. So we consider only the two nearer values of 23 which we get by incrementing or decrementing certain amount from 23 and these are 28 in forward direction and 20 in backward direction. Among these two 20 is more nearer than 28 because $23-20=3$ whereas $28-23=5$. So 23 is replaced with 20 instead of 28 . Some mathematical rules are discussed below by which the selection is done.

First the cover pixel (p) is divided by 8 and the remainder is found out. Then the remainder value is compared with target octal digit and the forward and backward differences from these two values are calculated by the following equation:

if the remainder ( $r$ ) of cover pixel (p) is less than or equal to the target octal digit (d) i.e. $\mathrm{r}<=\mathrm{d}$ then Forward Difference $\mathrm{f}:=\mathrm{d}-\mathrm{r}$

and Backward Difference $b:=r+8-d$

Otherwise i.e. if $r>d$ then

Forward Difference $\mathrm{f}:=\mathrm{d}+8-\mathrm{r}$

and Backward Difference $\mathrm{b}:=\mathrm{r}-\mathrm{d}$

For example if we consider 23 as cover pixel value (p) and 4 as target octal digit then $\mathrm{r}:=23 / 8:=7$ and $\mathrm{d}:=4$

i.e. $r>d$ so Forward Difference $f:=d+8-r=4+8-7=5$ and Backward Differences $\mathrm{b}:=\mathrm{r}-\mathrm{d}:=7-4:=3$.

Now by comparing forward and backward differences the nearest value of cover pixel whose remainder is equal to the target octal digit is decided. That is done by the following steps:

if Forward Difference (f) is less than Backward Difference (b) then the cover pixel value is incremented $\mathrm{f}$ decimal places so that its remainder becomes $\mathrm{d}$ and the modified value then becomes the corresponding stego pixel value (sp) i.e.

if $\mathrm{f}<\mathrm{b}$ then $\mathrm{sp}:=\mathrm{p}+\mathrm{f}$ and flag: $=1$ (flag used for $\mathrm{f}=\mathrm{b}$ case)

if Forward Difference (f) is greater than Backward Difference (b) then the cover pixel value is decremented b decimal places 
so that its remainder becomes $d$ and the modified value then becomes the corresponding stego pixel value (sp) i.e..

if $\mathrm{f}>\mathrm{b}$ then $\mathrm{sp}:=\mathrm{p}-\mathrm{b}$ and flag: $=0$ (flag used for $\mathrm{f}=\mathrm{b}$ case)

if Forward Difference (f) is equal to Backward Difference (b) then either the cover pixel value is incremented $\mathrm{f}$ decimal places or decremented $b$ decimal places based on the change of previous cover pixel value. The modified value then becomes the corresponding stego pixel value (sp) i.e.

if $\mathrm{f}=\mathrm{b}$ then $\mathrm{sp}:=\mathrm{p}+\mathrm{f}$ when flag $=1$

Otherwise $\mathrm{sp}:=\mathrm{p}-\mathrm{b}$ when flag $=0$.

The Forward (f) and Backward (b) Differences of previous example are 5 and 3 . The cover pixel (p) value is 23 .

Since $\mathrm{f}>\mathrm{b}$ then stego pixel value $\mathrm{sp}$ is $20(\mathrm{sp}:=\mathrm{p}-\mathrm{b}:=23-$ $3:=20)$ and flag: $=0$.

Boundary value of the pixels creates some problem in our proposed method. The problems are shown in the following table.

Table 1: Problem with Boundary Value of the Pixel

\begin{tabular}{|c|c|c|c|c|c|c|c|c|}
\hline 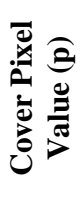 & 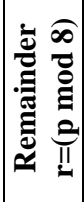 & 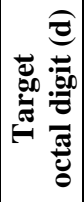 & 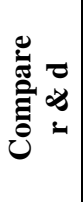 & 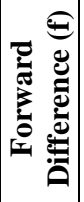 & 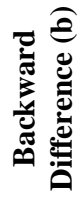 & ڤ & 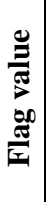 & 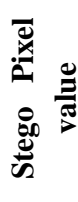 \\
\hline 0 & 0 & 5 & $\mathrm{r}<\mathrm{d}$ & 5 & 3 & $f>b$ & 0 & $\begin{array}{l}-3 \\
(253)\end{array}$ \\
\hline 254 & 6 & 1 & $\mathrm{r}>\mathrm{d}$ & 3 & 5 & $\mathrm{f}<\mathrm{b}$ & 1 & $\begin{array}{l}257 \\
(1)\end{array}$ \\
\hline 255 & 7 & 3 & $\mathrm{r}>\mathrm{d}$ & 4 & 4 & $f=b$ & 1 & $\begin{array}{l}259 \\
\text { (3) }\end{array}$ \\
\hline 2 & 2 & 7 & $\mathrm{r}<\mathrm{d}$ & 5 & 3 & $f>b$ & 0 & $\begin{array}{l}-1 \\
(255)\end{array}$ \\
\hline
\end{tabular}

From Table 1 it is seen that if the actual replacement rule on cover image is followed then the boundary value gives the high distortion in the stego image. Here this problem is also solved by considering some rule. That is if the modified pixel value is less than 0 or greater than 255 by either considering forward or backward differences, then the cover pixel value is replaced by the other difference i.e. backward and forward differences. That is explained by showing the result of the Table 1 in the Table 2.

Table 2: Solution for Boundary Value Problem

\begin{tabular}{|c|c|c|c|c|c|}
\hline 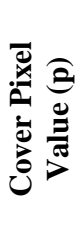 & 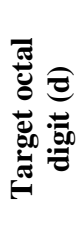 & 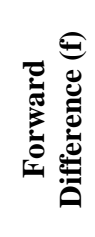 & 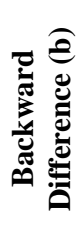 & 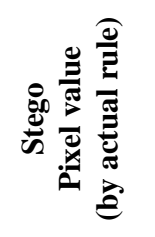 & 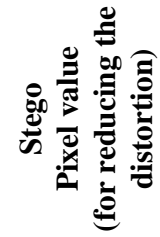 \\
\hline 0 & 5 & 5 & 3 & -3(i.e. 253) & $0+5=5$ \\
\hline 254 & 1 & 3 & 5 & 257 (i.e. 1) & $254-5=249$ \\
\hline 255 & 3 & 4 & 4 & 259 (i.e. 3) & $255-4=251$ \\
\hline 2 & 7 & 5 & 3 & -1 (i.e. 255$)$ & $2+5=7$ \\
\hline
\end{tabular}

\subsubsection{Storing the Length of Target Text}

Now for extraction it is need to store string length within the cover image. The string length is stored as string instead of number. The technique is divided into two parts: first storing the length of string length, then the storing of actual string length as a string.

The Length of string length is calculated from number of digits present within string length. Then this value stored at the first row of cover pixel using standard LSB Technique.

Let "Image" is target data then the length of it is 5 . Number of digit within 5 is 1 so the length of the target string length is 1 . This value is now converted in 8 bit decimal i.e. 00000001 . These 8 bits are now embedded at the first row of cover media using LSB technique.

The string length is stored at the last row of cover image using the proposed remainder technique. For this the length of string should be multiples of three. If not then it is necessary to make it multiple of three. From the above example the string length is " 5 ". Now this " 5 " is considered as a string instead of number for storing and pass through preprocessing stage. Then remainder method is used to store it.

\subsection{Data extraction}

The decoding method at the receiver side also divided into three major steps: extraction of data using Remainder method, Post-processing of data and before this two steps length extraction.

\subsubsection{Target string length extraction}

First the length of string length is extracted from 1st row of stego image. Then the actual string length is extracted from the last row of the stego media using the remainder method for extraction and post processing which are discussed below.

\subsubsection{Remainder method for Extraction}

At the receiver side the affected (where the data is hidden) pixel values are divided by 8 and the remainders of this division are hidden octal string of Preprocess step of sender side. Now this is send to the post-processing technique to get the original target text.

Suppose $\left[\begin{array}{lllllllllllll}\ldots & 20 & 44 & 167 & 59 & 74 & 1 & 118 & 243 & 151 & 73 & 186 & 12\end{array}\right.$ ...] these are the affected pixel value of stego image on which the remainder method for extraction is applied. These modified pixel values of cover image are now divided by 8 and remainders are $\left[\begin{array}{llllllllllll}4 & 4 & 7 & 3 & 2 & 1 & 6 & 3 & 7 & 1 & 2 & 4\end{array}\right]$. This is the octal string that is generated from the original target data by preprocessing technique used at the time of sender side encoding. Now this string is send to post-processing technique to get the original target text at the receiver side.

\subsubsection{Post-processing}

The resultant octal string is the input to this stage. Here each octal digit is converted to their 3 bit binary equivalent. Each of these 3 bits is concatenated to form a binary string. Then every 7 bits are cut and converted to their corresponding decimal equivalent. Then the characters of these ASCII values are concatenated up to the extracted string length to get the original target string.

Let the input octal string is $\left[\begin{array}{lllllllllllll}4 & 4 & 7 & 3 & 3 & 4 & 1 & 6 & 3 & 7 & 1 & 2 & 4\end{array}\right.$ 7]

The 3 bit binary equivalent of these digits are $\left[\begin{array}{llll}100 & 100 & 111\end{array}\right.$ $\begin{array}{lllllllllll}011 & 011 & 100 & 001 & 110 & 011 & 111 & 001 & 010 & 100 & 111]\end{array}$ 
After concatenating

[100100111011011100001110011111001010100111] this

binary string is formed.

Now this are grouped into 7 bit each $-[10010011101101$ $1100001110011111001010100111]$

Their Corresponding ASCII are [ [ [ $\left.73 \begin{array}{lllll}109 & 97 & 103 & 101 & 39\end{array}\right]$

According to string length of target text it is 5 so the first 5 ASCII values are consider for Target string [ [ $\begin{array}{llll}73 & 109 & 97 & 103\end{array}$ 101].

Now the Target string is "Image".

\section{ALGORITHM FOR PROPOSED METHOD}

\subsection{Algorithm for embedding target text within a cover image}

Step 1: Start

Step 2: Read the target text file and Cover image.

Step 3: Store the length of the target text.

Step 4: Preprocess the Target text.

Step5: Embed preprocessed target text from the second row of the cover image matrix using remainder method

Step 6: Send the obtained Stego Image to the receiver. Step 7: End

\subsubsection{Storing the Length of the Target Text}

Step 1: Hide the length of target string length using LSB technique.

Step 2: Preprocess the string length.

Step 3: Then hide preprocessed string length from the last element of the last row of the cover image matrix using remainder method.

Step 4: Return

\subsubsection{Preprocessing}

Step 1: Make the length of input string as a multiple of 3.

Step 2: Convert ASCII of each Character into 7 bit binary.

Step 3: Group the array elements into 3 bits each and convert them to their corresponding Octal equivalents.

Step 4: Return

\subsubsection{Proposed Remainder Method}

Step 1: Read a pixel value of the cover image matrix and find modulus 8 of this pixel.

Step 2: Consider a digit of target octal string.

Step 3: Calculate Forward and Backward Differences.

Step 4: Compare them and decide the adjusted value.

Step 5: Modify the cover pixel value with this adjusted value.

Step 6: repeat above steps for the length of the octal string.

Step 7: Return

\subsection{Algorithm for extracting target text from a Stego image}

Step 1: Read the stego image matrix

Step 2: Extract the string length.

Step 3: Extract the target octal string using modulo operator.

Step 4: Post-process the extracted octal string to get the Step 5: End original target string.

\subsubsection{Eextraction of string length}

Step 1: Extract the length of the string length, from the LSB of first 8 pixels of Stego Image.

Step 2: Extract string length using remainder method for extraction
Step 3: Post-process the extracted string length to get the actual string length.

Step 4: Return

\subsubsection{Extracting target data using proposed Remainder method}

Step 1: Calculate Octal String length

Step 2: Read a pixel value of the stego image matrix and find modulo 8 of this pixel.

Step 3: This remainder is the embedded octal digit

Step 4: Repeat step 2 and 3 for the length of the octal string.

Step 5: Concatenate remainders to get the target octal string.

Step 6: Return.

\subsubsection{Post-processing}

Step 1: Convert each digit of Octal string into 3 bit binary and concatenate to get a binary String.

Step 2: Group the bits of this string, 7 at a time, and convert each of these groups into their corresponding decimal value to get the ASCII equivalents.

Step 3: Repeat the above steps for the string length.

Step 4: Convert the ASCII value to get the original target string.

Step 5: Return

\section{RESULT \& ANALYSIS}

In this section first we show the experimental results of the proposed technique in Fig. 1. Then the analysis of the proposed technique is done with existing relevant methods based on quality and capacity analysis of cover media. Also a standard benchmark tool - StirMark Benchmark is used for performance analysis of proposed technique.

Target text is chosen for embedding into the cover image are of three types based on their size. Text file with no. of characters less than 100 is considered to be small. Text file with no. of characters in the range of 100-500 is considered to be medium. Text file with no. of characters greater than 500 is considered to be large. As a cover image we consider uncompressed gray scale image file of different size. The images are including landscapes, people, jungles, animals, motion, single object image and many more.

\subsection{Analysis based on image quality}

The image quality of the output stego images are analyzed by histogram analysis, MSE and PSNR calculation, Euclidian distance measurement

\subsubsection{Histogram analysis}

An image histogram acts as a graphical representation of the tonal distribution in a digital image. It plots the number of pixels for each tonal value. From Fig. 1 we observe that there are no significant changes in frequency of appearance of the gray shades by comparing the histogram of cover image with the stego image.

\subsubsection{PSNR and MSE calculation}

The PSNR (Peak Signal to Noise Ratio) is used to measure the quality of stego image compared to the cover image and is calculated by equation (1), where MSE defined in equation (2) refers to Mean Square Error.

$$
\begin{aligned}
& P S N R=10 \log \left(\left(\frac{255^{2}}{M S E}\right)\right) d b \\
& M S E=\frac{1}{m n} \sum_{i=1}^{m} \sum_{j=1}^{n}[C(i, j)-S(i, j)]^{2}
\end{aligned}
$$


Where $\mathrm{m}, \mathrm{n}$ are the width and height of Cover (C) and Stego (S) image matrix.

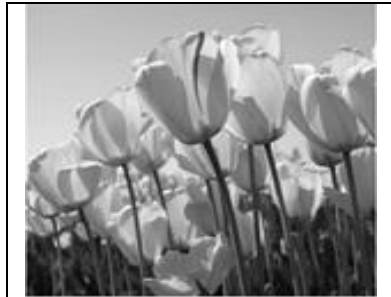

(a) Tulip

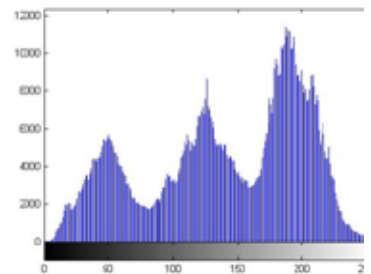

(c)

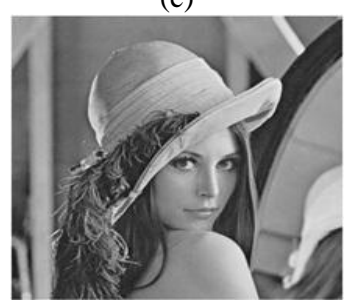

(e) Lenna

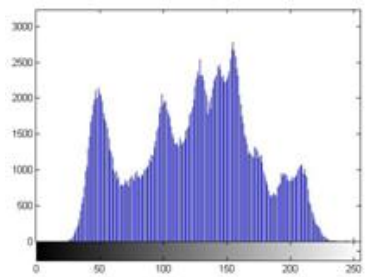

(g)

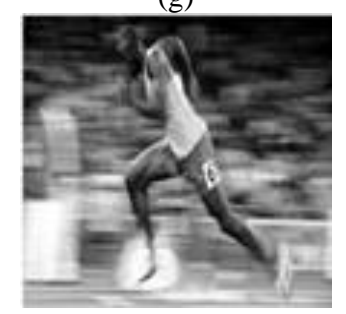

(i) Motion

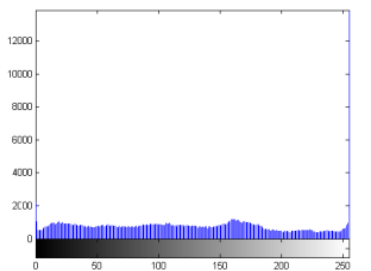

(k)

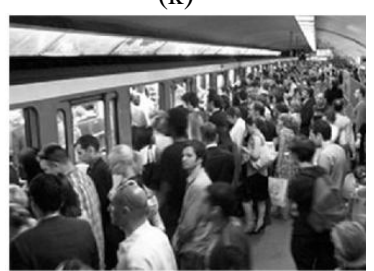

(m) Crowdy (b) Tulip Stego

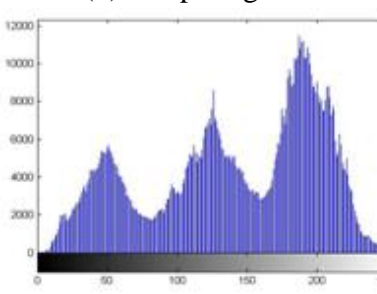

(d)

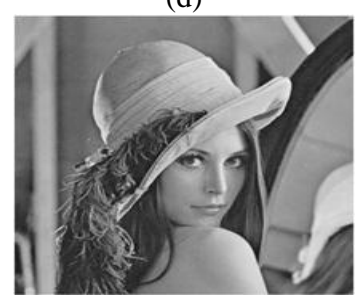

(f) Lenna Stego

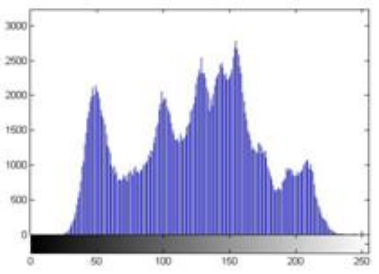

(h)

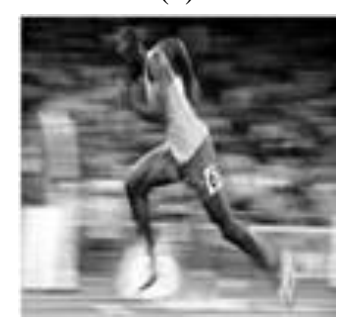

(j) Motion Stego

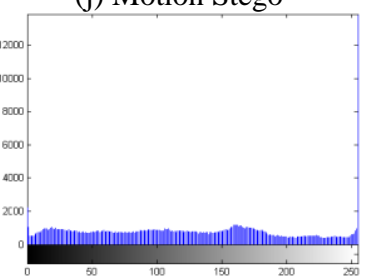

(1)

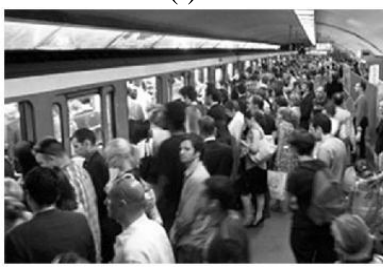

(n) Crowdy Stego

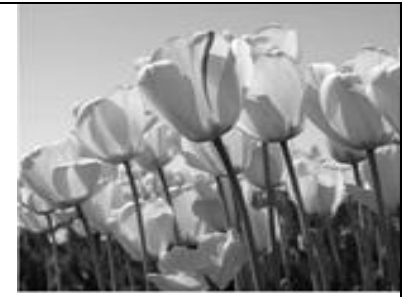

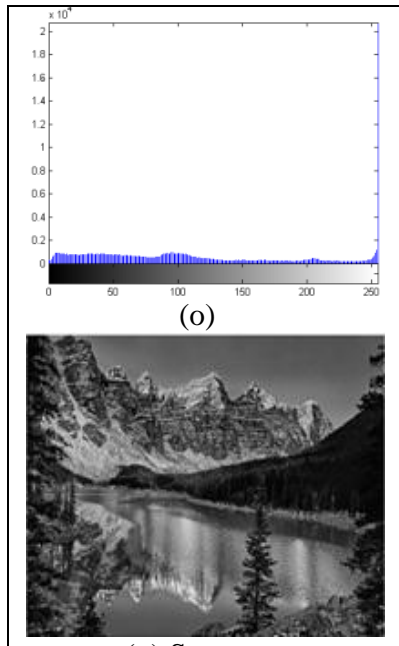

(q) Scenery

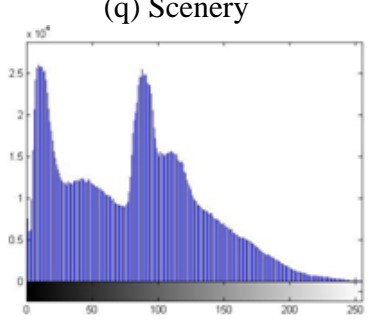

(s)

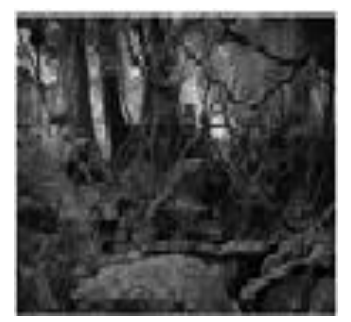

(u) Jungle

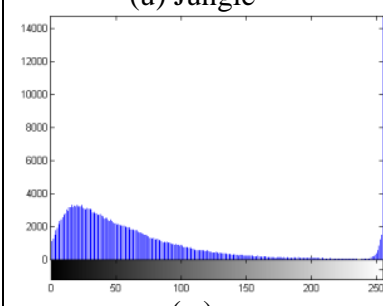

(w)

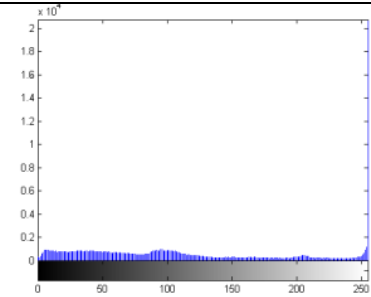

(p)

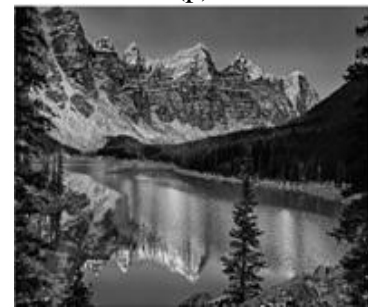

( r) Scenery Stego

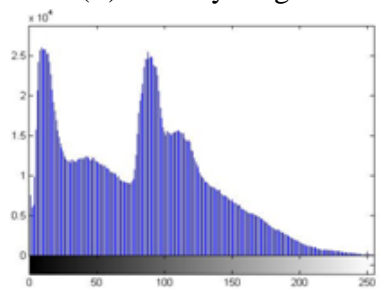

(t)

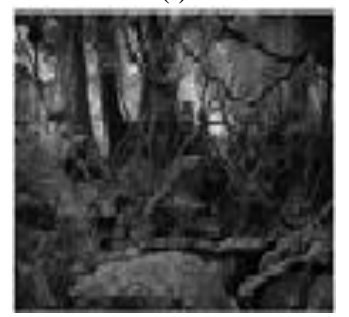

(v) Jungle Stego

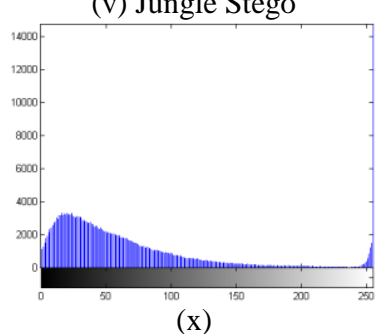

(x)

Fig.1: Experimental Result. (b), (f), (j), (n), (r) and (v) are the stego images of (a), (e), (i), (m), (q) and (u). (c), (g), (k), (o), (s) and (w) are histogram of the cover images. (d), (h), (l), (p), (t) and (x) are the histogram of corresponding stego images.

The quality of the image is higher if the PSNR value of the image is high. Since PSNR is inversely proportional to MSE value of the image, the higher the PSNR value is lower the MSE value will be. The better stego image quality is lower the MSE value and higher PSNR will be. Fig. 2 and Fig. 3 show the MSE and PSNR values when same text embedded within different cover image. Whereas Fig. 4 and Fig. 5 show the same for different target text embedded within same cover image. 


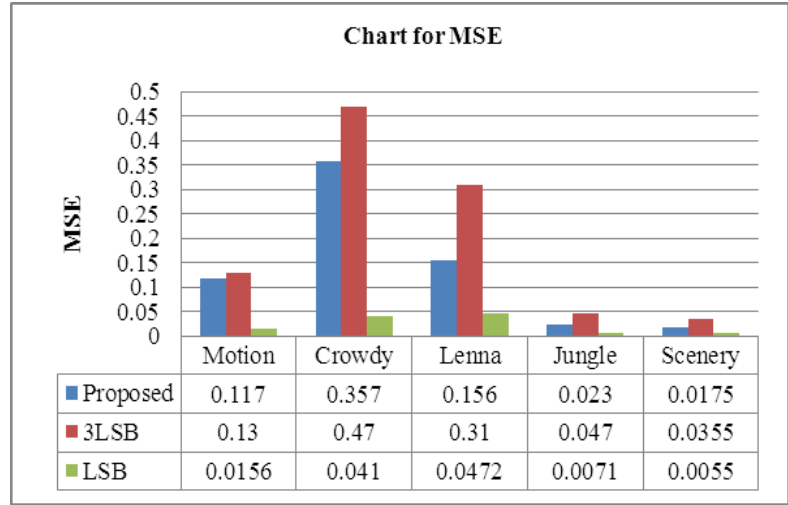

Fig 2: MSE for Same Target Text Embedded in Different Cover Image

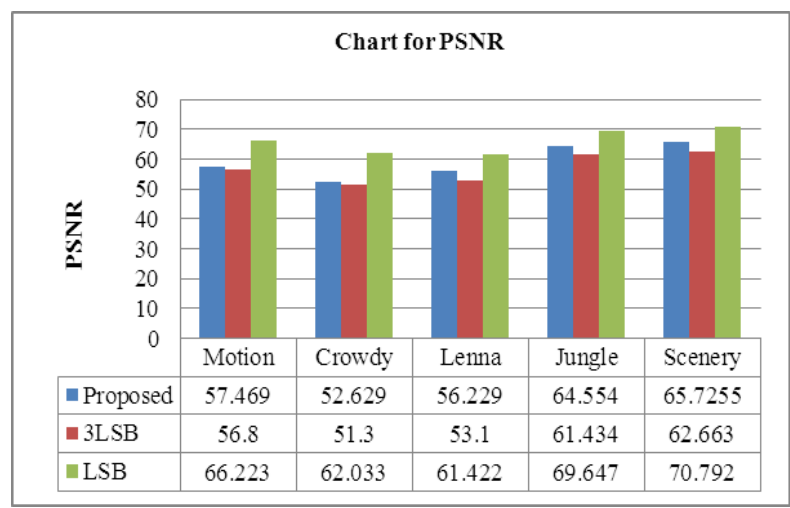

Fig 3: PSNR for Same Target Text Embedded in Different Cover Image

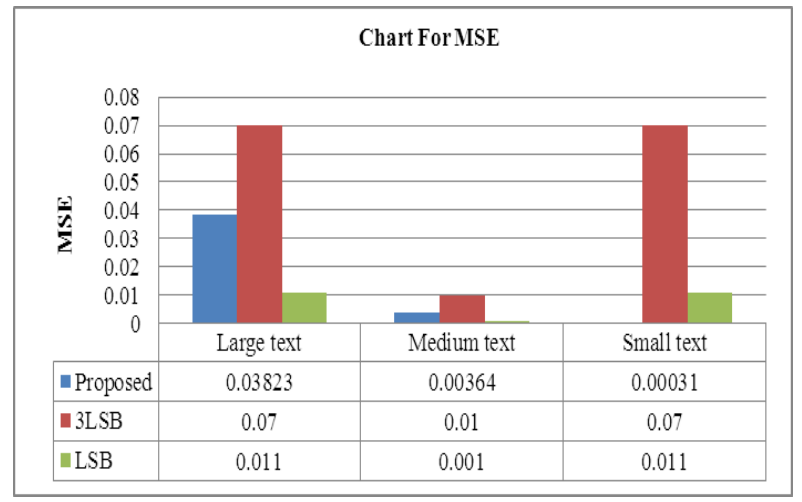

Fig 4: MSE Different Size Target Text Embedded in Same Cover Gray Scale Image

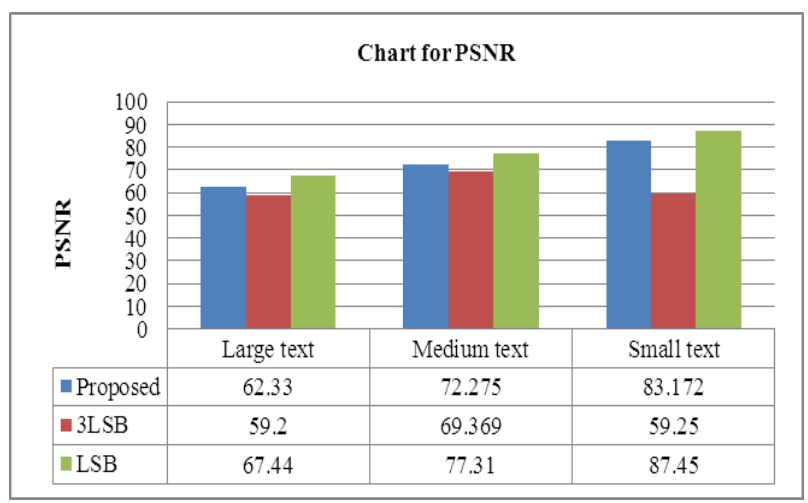

Fig 5: PSNR Different Size Target Text Embedded in Same Cover Gray Scale Image

\subsubsection{Calculation of Euclidian distance of image}

The Euclidean distance (d) of the cover and stego images is used to show that there is not much change in the intensity perception of the two images. The objective of this measure is to perceptibility of proposed technique. If the Euclidean distance is very high then there is a possibility that pixels have been changed more with its intensity.

$d=\sqrt{\left(c_{1}-c_{2}\right)^{2}}$

Where $\mathrm{c} 1$ and $\mathrm{c} 2$ are the pixel values of the cover and the stego image respectively.

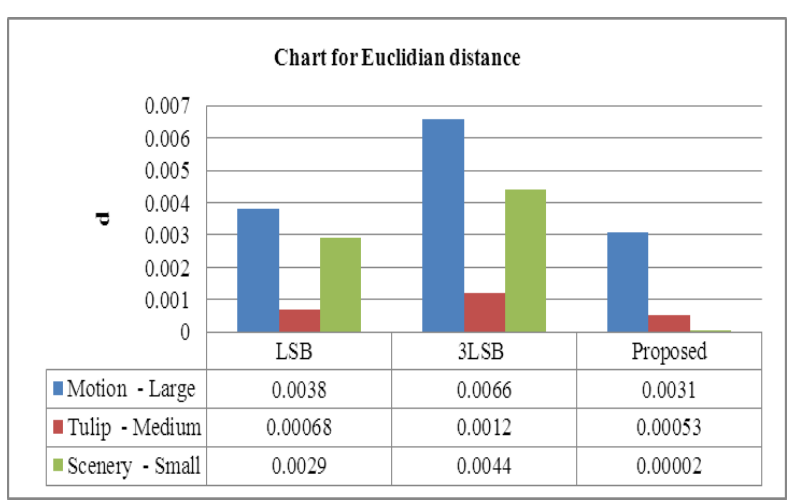

Fig 6: Euclidian distance Measurement

\subsubsection{Analysis based on StirMark benchmark 4.0}

StirMark Benchmark Version 4.0 was introduced on January, 2012 for the fair evaluation procedures for steganography. Different tools like Embedded/Extraction time, Add noise, Median Cut, Self-similarities, Convolution filter, cropping, rotation, PSNR and many more are used for better analysis of stego image with its cover image. Now we analyze the efficiency of our proposed technique based on this standard benchmark tool and the corresponding results are shown in Table 3.

\subsubsection{Embedding Capacity of Cover image}

We analyze capacity of cover media based on the number of target bits embedded within that media. Capacity is again inversely proportional to robustness as well as perceptibility. So our aim is to increase capacity without any noticeable distortion. We also compare our proposed technique with both LSB and 3LSB technique to so efficiency of it.

From Table 4 it is observed that provide more capacity than standard LSB technique. In case of standard LSB minimum c * 7 number of pixels are required to store c different characters whereas for 3LSB multi-bit and our Proposed Remainder Technique it is $\mathrm{c}^{*} 7 / 3$. Since in Multi bit 3LSB technique 3 different target bits are embedded into a single pixel and in Proposed Technique 3 different bits of target text are used for creating octal digit. Similarly in the case of our proposed technique and that is embedded into a cover pixel. That's why Embedding Capacity of a Cover image using 3LSB and our proposed method is 3 times more than that of using Standard LSB technique.

Our proposed technique is better than 3LSB multi-bit steganography in respect to perceptibility but not than standard LSB technique. In the case of LSB single bit embedded at the LSB of cover pixel and the maximum error cause for this embedding is $2^{1}-1$ i.e. 1 intensity value. In the case of 3LSB multi-bit steganography three Least Significant 
Table 3: StirMark Benchmark Results

\begin{tabular}{|c|c|c|c|c|c|c|c|c|c|c|c|c|c|}
\hline 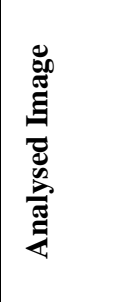 & 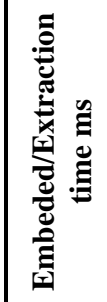 & 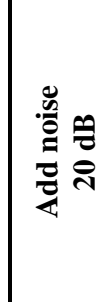 & 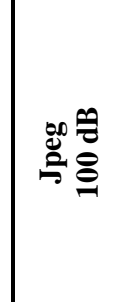 & 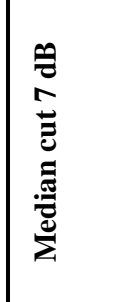 & 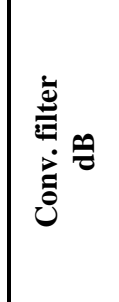 & 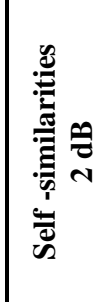 & 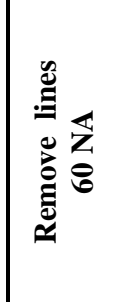 & 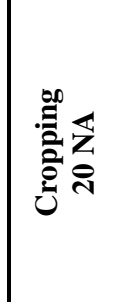 & 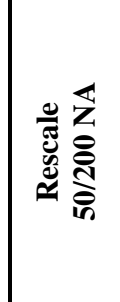 & 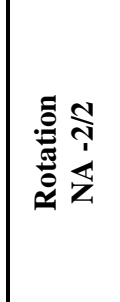 & 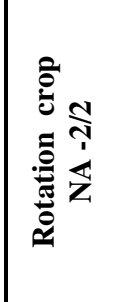 & 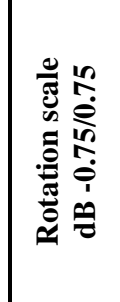 & 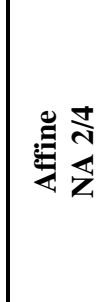 \\
\hline \multirow{2}{*}{ Lenna } & \multirow{2}{*}{21} & \multirow{2}{*}{6.4379} & \multirow{2}{*}{62.7552} & \multirow{2}{*}{29.1634} & 11.7828 & \multirow{2}{*}{2.0407} & \multirow{2}{*}{178.548} & \multirow{2}{*}{143.784} & 178.04 & 167.207 & 172.946 & 19.9198 & 169.26 \\
\hline & & & & & -6.0077 & & & & 178.409 & 167.205 & 172.946 & 20.7387 & 171.25 \\
\hline \multirow{2}{*}{$\begin{array}{l}\text { Lena } \\
\text { stego }\end{array}$} & \multirow{2}{*}{7} & \multirow{2}{*}{6.4383} & \multirow{2}{*}{62.7486} & \multirow{2}{*}{29.1658} & 11.7825 & \multirow{2}{*}{2.5284} & \multirow{2}{*}{178.537} & \multirow{2}{*}{143.784} & 178.005 & 167.188 & 172.937 & 19.9163 & 169.25 \\
\hline & & & & & -6.0073 & & & & 178.391 & 167.187 & 172.936 & 20.7348 & 171.24 \\
\hline \multirow{2}{*}{ Crowdy } & \multirow{2}{*}{21} & \multirow{2}{*}{6.3435} & \multirow{2}{*}{63.3793} & \multirow{2}{*}{26.4385} & 14.7354 & \multirow{2}{*}{1.5488} & \multirow{2}{*}{182.686} & \multirow{2}{*}{90.5154} & 182.743 & 170.622 & 175.885 & 16.802 & 171.12 \\
\hline & & & & & -6.502 & & & & 182.703 & 170.626 & 175.887 & 17.6868 & 176.94 \\
\hline \multirow{2}{*}{$\begin{array}{l}\text { Crowdy } \\
\text { stego }\end{array}$} & \multirow{2}{*}{5.8} & \multirow{2}{*}{6.3235} & \multirow{2}{*}{63.3717} & \multirow{2}{*}{26.4384} & 14.7354 & \multirow{2}{*}{1.5489} & & & 182.74 & 170.624 & 175.876 & 16.802 & 171.11 \\
\hline & & & & & -6.502 & & 102.004 & & 182.703 & 170.624 & 175.878 & 17.6868 & 176.93 \\
\hline & & & & & 14.0442 & & & & 120.931 & 112.426 & 105.622 & 14.0943 & 111.93 \\
\hline Juingic & & 0.4100 & p1.104t & $20.22>0$ & -1.047 & P.गय2 & 120.020 & 0.2020 & 120.641 & 112.45 & 105.62 & 14.0072 & 117.54 \\
\hline Jungle & & & & & 14.0442 & & & & 120.926 & 112.466 & 105.628 & 14.0951 & 111.93 \\
\hline stego & & & & & -1.047 & & & & 120.64 & 112.451 & 105.628 & 14.0082 & 117.54 \\
\hline M. & & 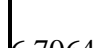 & L & ho 967 & 13.0876 & h & $\mathrm{J}_{160}$ & & 167.951 & 157.229 & 160.115 & 18.2827 & 157.60 \\
\hline (1) & PJ & 0.1704 & 02.3421 & 29.201 & -4.9511 & $1-2231$ & 100.400 & 120.010 & 168.217 & 157.222 & 160.116 & 18.2588 & 162.84 \\
\hline Motion & $58+2$ & 67063 & G2 3151 & 2667 & 13.0871 & b 2221 & 168410 & 12050 & 167.966 & 157.247 & 160.126 & 18.2995 & 157.62 \\
\hline stego & & 0.700 & (1) & F-2000 & -4.9512 & & 100.419 & 120.59 & 168.229 & 157.239 & 160.128 & 18.2855 & 162.86 \\
\hline Scenery & $\mathrm{bog}$ & b 3444 & 62110 & bo 5226 & 12.3456 & 34047 & 140006 & & 148.935 & 138.935 & 137.428 & 14.7271 & 138.13 \\
\hline text) & 20 & $\left.\right|^{\prime .5444}$ & 02.119 & 20.5220 & -1.7801 & p.4047 & 149.090 & 109.4005 & 149.123 & 138.924 & 137.429 & 14.6611 & 145.15 \\
\hline Scenery & 16 & h 2224 & 0000 & & 12.3455 & 2000 & 110 & & 148.935 & 138.936 & 137.413 & 14.7275 & 138.13 \\
\hline stego & +.0 & | & 02.0900 & 20.0225 & -1.7801 & 2.907 & 147.092 & 09.4005 & 149.125 & 138.929 & 137.412 & 14.6615 & 145.15 \\
\hline Tulip & bo & 61476 & 633834 & bo 1522 & 13.1066 & 1508 & 180126 & 154833 & 188.545 & 177.089 & 184.287 & 20.4316 & 179.26 \\
\hline Text) & $=0$ & $0.14 / 0$ & 00.0034 & 29.1532 & -6.4529 & 1.590 & 109.120 & 104.053 & 188.995 & 177.089 & 184.288 & 20.2285 & 181.37 \\
\hline Tulip & & & & & 13.1066 & & & & 188.556 & 177.082 & 184.302 & 20.4314 & 179.26 \\
\hline stego & 4.4 & $0.14 / 0$ & 03.0834 & 29.1522 & -6.4529 & 1.598 & 189.124 & (154.053 & 188.993 & 177.08 & 184.303 & 20.2283 & 181.37 \\
\hline
\end{tabular}

Table 4. Capacity of Different Gray Scale Images to Embed Different Target Text

\begin{tabular}{|c|c|c|c|c|c|c|c|c|}
\hline 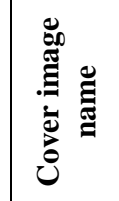 & 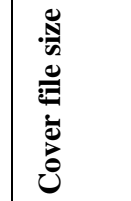 & 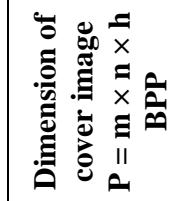 & 莺 & 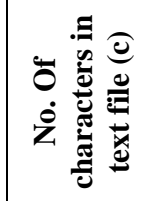 & 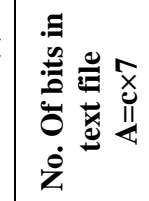 & 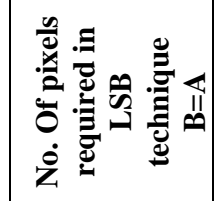 & 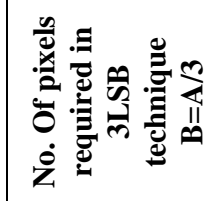 & 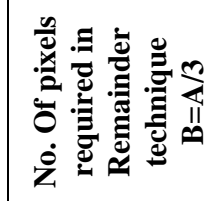 \\
\hline Motion & $2.69 \mathrm{MB}$ & $1024 * 768 * 8$ & 3.05KB (large) & 3130 & 21910 & 21910 & 2435 & 2435 \\
\hline Tulip & $3.80 \mathrm{MB}$ & $1024 * 1024 * 8$ & 332 bytes(medium) & 322 & 2254 & 2254 & 251 & 251 \\
\hline Scenery & $7.36 \mathrm{MB}$ & $1920 * 1200 * 8$ & 22 bytes (small) & 22 & 154 & 154 & 18 & 18 \\
\hline
\end{tabular}

bits are affected together by 3 bits of target data and the maximum error cause for this embedding is $2^{2}+2^{1}+2^{0}$ i.e 7 .

But in the case of our proposed technique the maximum change is 4 . Since the cover pixel is divided by 8 the eight different remainder values of the range 0 to 7 are possible as well as 0 to 7 different octal digits are possible since the radix of octal number system is 8 . Also the minimum of forward and 
backward differences is considered then the change of cover pixel value after adjustment is never greater than four. The fig 7 helps us to understand why the change of cover pixel is maximum 4 .

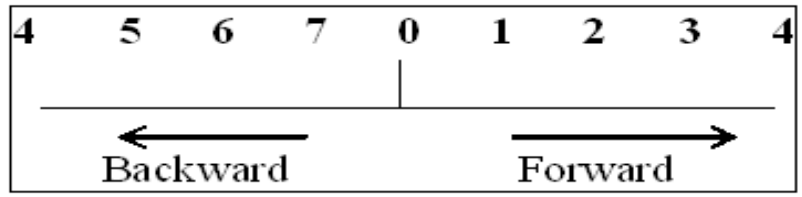

Fig: 7 Perceptibility Measurements

Our Proposed Technique is robust in the sense that if anyone can able get embedded target data from stego media they do not get the original target text without knowing the post-processing technique; they just get the octal string.

\section{CONCLUSION}

This paper proposes a high quality steganography technique using remainder method. Here the overlapping problem of remainder is solved by adjusting pixel values with minimum distortion. Also the preprocessing step for target data provides more robustness to this propose method. Experimental result also compared with the standard LSB technique and 3LSB multi-bit technique. It shows better performance than 3LSB multi-bit steganography in respect to imperceptibility. Although with respect to capacity the multi-bit technique as well as our proposed technique is same but is better than standard LSB technique. Experimental results also tested with respect to StirMark Benchmark version 4.0 and give satisfactory results.

\section{REFERENCES}

[1] Diffie, W. and Hellman, M. E. 1977. "Exhaustive Cryptanalysis of the NBS Data Encryption Standard". IEEE Computer, Vol. 10, pp. 74- 84

[2] Provos, N., Honeyman, P. 2003. "Hide and seek: An introduction to steganography". IEEE Security \& Privacy Magazine 1, pp. 32-44.

[3] Anderson, R. J., Petitcolas, F. A. P., "On the limits of steganography", IEEE Journal of selected Areas in Communications, May 1998.

[4] Jamil, T. 1999. "Steganography: The art of hiding information is plain sight", IEEE Potentials, Vol. 18, No. 01 .

[5] Silman, J. 2001. "Steganography and Steganalysis: An Overview". SANS Institute.

[6] Johnson, N. F., Jajodia, S., "Exploring Steganography: Seeing the Unseen", Computer Journal, Feb. 1998.

[7] Zhang, X., Wang, S., Zhou, Z. 2008. "Multibit Assignment Steganography in Palette Images". IEEE Signal Processing Letters, Vol. 15.
[8] Luo, W., Huang, F., Huang, J. "Edge Adaptive Image Steganography Based on LSB Matching Revisited". IEEE Transactions on Information Forensics And Security, Vol. 5, No. 2, Jun. 2010.

[9] Kurak, C., McHugh, J., "A Cautionary Note on Image Downgrading". In Proc. of IEEE 8thAnnual Computer Security Applications Conference. San Antonio, USA, Nov./Dec. 1992, pp. 153-155.

[10] Chan, C. K., Cheng, L. M., "Hiding data in images by simple LSB substitution", Pattern Recognition, pp. 469474, Mar. 2004.

[11] Wang, R. Z., Lin, C. F., Lin, J. C., "Image hiding by optimal LSB substitution and genetic algorithm". Pattern Recognition 34, pp.671-683, Mar. 2001.

[12] Roque, J. J., Minguet, J. M. 2009. "SLSB: Improving the Steganographic Algorithm LSB". Workshop on Security in Information Systems WOSIS

[13] Kumari, L., Goyal, D., Gyan, S. "Analysis and Design of 3 LSB Techniques for Secure Audio Steganography". International Journal of Advanced Research in Computer Engineering \& Technology (IJARCET) Vol. 2, No. 8, Aug. 2013.

[14] Nguyen B. C., Yoon S. M., Lee H. K. 2006. "Multi Bit Plane Image Steganography". IWDW, LNCS 4283, pp. 61-70.

[15] Tripathi A., Nair J. S. "Steganography Using Segmenting Mosaic Images with Embedding Data by 3 LSB S-Type Scanning". International Journal of Latest Trends in Engineering and Technology (IJLTET) Vol. 2, No. 4 Jul. 2013.

[16] Zhang X., Wang S., Zhou Z. 2008. "Multibit Assignment Steganography In Palette Images". IEEE Signal Processing Letters, Vol. 15.

[17] Kawaguchi E., Eason R. O. "Principle and applications of bpcs steganography". In Proc. of SPIE 3528, Multimedia Systems and Applications Conference Vol. 3528, Nov. 01, 1998.

[18] Thien, C. C., Lin, J. C. "A simple and high-hiding capacity method for hiding digit-by-digit data in images based on modulus function". Pattern Recognition 36, 2875-2881, Dec. 2003.

[19] Wang, C.M., Wu, N. I., Tsai, C. S., Hwang, M. S. 2008 "A high quality steganographic method with pixel-value differencing and modulus function". The Journal of Systems and Software, pp. 150-158.

[20] Wang, S.J., "Steganography of capacity required using modulo operator for embedding secret image", Applied Mathematics and Computation 164, pp. 99-116, May. 2005. 